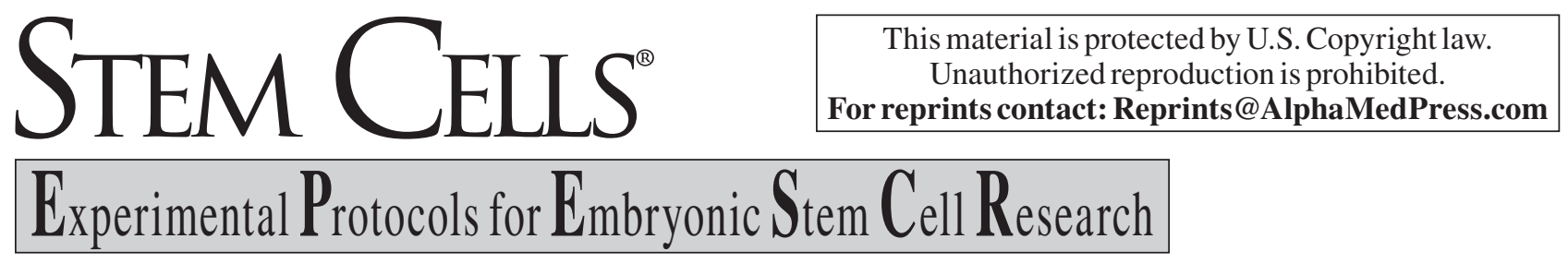

\title{
Methods for Derivation of Human Embryonic Stem Cells
}

\author{
Hee Sun Kim, ${ }^{\mathrm{a}, \mathrm{b}}$ Sun Kyung Oh, ${ }^{\mathrm{a}, \mathrm{b}}$ Yong Bin Park, ${ }^{\mathrm{b}, \mathrm{c}}$ Hee Jin Ahn, ${ }^{\mathrm{b}}$ Ki Cheong Sung, ${ }^{\mathrm{a}}$ Moon Joo Kang, ${ }^{\mathrm{a}}$ \\ Lim Andrew Lee, ${ }^{\mathrm{b}}$ Chang Suk Suh, ${ }^{\mathrm{a}, \mathrm{b}, \mathrm{d}}$ Seok Hyun Kim, ${ }^{\mathrm{a}, \mathrm{b}}$ Dong-Wook Kim, ${ }^{\mathrm{e}}$ Shin Yong Moon ${ }^{\mathrm{a}, \mathrm{b}}$
}

\begin{abstract}
aDepartment of Obstetrics and Gynecology, 'Institute of Reproductive Medicine and Population, Medical Research Center, College of Medicine, Seoul National University, Seoul, Korea; 'Central Research Institute, Sam Jin Pharm. Co. Ltd., Hwasung, Korea; ${ }^{\mathrm{d} D e p a r t m e n t ~ o f ~ O b s t e t r i c s ~ a n d ~ G y n e c o l o g y, ~ S e o u l ~ N a t i o n a l ~ U n i v e r s i t y ~ B u n d a n g ~ H o s p i t a l, ~}$ Seongnam, Korea; ${ }^{\mathrm{e}}$ Department of Physiology, Yonsei University College of Medicine, Seoul, Korea
\end{abstract}

Key Words. Embryonic stem cells • Derivation - Immunosurgery • Partial-embryo culture Whole-embryo culture • Blastocyst quality • Inner cell mass

\begin{abstract}
The expanded blastocysts, developed from 2PN-stage embryos, are generally divided into three categories: a good blastocyst containing a large and distinguishable inner cell mass (ICM), a blastocyst with a small and distinct ICM, and a blastocyst with a poorly defined ICM. In this study, we introduce methods for the derivation of human embryonic stem cells (hESCs) depending on the quality of the blastocysts. An immunosurgical method was used for the good expanded blastocysts. This method, however, raises the probability of ICM loss in cases of hESC derivation from blastocysts with smaller or indistinct ICMs. Furthermore, this method is also associated with a risk of the contamination of the hESCs with animal pathogens. To overcome these shortcomings, the partial- or
\end{abstract}

whole-embryo culture method was used. For blastocysts with no visible ICM, the whole-embryo culture method was used to establish hESCs via the seeding of the entire blastocyst without its zona pellucida directly on a STO feeder layer. However, trophectodermal overgrowth tends to hinder the expansion of the ICM during the initial steps of hESC derivation. Therefore, the partial-embryo culture method was developed to establish hESCs from blastocysts with smaller ICMs. The surgical isolation of the region containing the ICM with an ultra-fine glass pipette alleviates trophectoderm overgrowth. This method is also applicable to blastocysts with large and distinct ICMs, and the efficiency of this method is comparable to that of the immunosurgical method. STEM CELLS 2005;23:1228-1233

\section{INTRODUCTION}

Embryonic stem cells (ESCs), in the absence of any specific differentiation conditions, will maintain their self-renewal for an indefinite period. These cells, which possess the enviable ability to differentiate into any possible cell type in the body, have become a primary focus of interest in the context of cell replacement therapy and have also garnered a great deal of attention in developmental or drug/toxin screening studies. However, to facilitate such studies, a comprehensive outline of efficient ESC derivation will be required.
Fourteen years after the initial isolation of ESCs from mouse blastocysts [1, 2], Thomson et al. [3] at the University of Wisconsin (Madison, WI) first established primate ESCs from the blastocysts of Rhesus monkeys. Then, in 1998, they were able to successfully derive human embryonic stem cells (hESCs) from human blastocysts [4]. Since then, the derivation of hESCs from the inner cell mass (ICM) of preimplantation blastocysts has become a procedure undertaken by many researchers all over the world [4-11].

Correspondence: Shin Yong Moon, M.D., Ph.D., Department of Obstetrics and Gynecology, College of Medicine, Seoul National University, 28 Yongon-dong, Chonno-gu, Seoul 110-744, Korea. Telephone: 82-2-2072-2384; Fax: 82-2-3672-7601; e-mail: shmoon@ plaza. snu.ac.kr; and Dong-Wook Kim, Ph.D., Department of Physiology, Yonsei University College of Medicine, 134 Shinchon-dong, Seodaemun-gu, Seoul 120-752, Korea. Telephone: 82-2-2228-1703; Fax: 82-2-393-0203; e-mail: dwkim2@ yumc.yonsei.ac.kr Received October 25, 2004; accepted for publication July 7, 2005; first published online in STEM CELLS EXPRESS July 28, 2005; available online without subscription through the open access option. @AlphaMed Press 1066/5099/2005/\$12.00/0 doi: 10.1634/stemcells.2004-0296

STEM CELLS 2005;23:1228-1233 www.StemCells.com 
There are currently several extant reports of hESC lines having been established in a variety of worldwide laboratories. However, a comprehensive hESC derivation method based on the quality of the blastocysts has yet to be definitively elucidated. The isolation of ICM by immunosurgery, a procedure that was developed by Solter and Knowles in 1975 [12], has become common practice in most laboratories in which ESC derivation takes place. However, according to our experience, immunosurgery may not be the method of choice in all cases. Not all embryos develop into the good expanded blastocysts, which are characterized by large, distinct ICMs. In some cases, the embryos are found to possess smaller ICMs. In many cases, blastocysts also possess poorly defined ICMs.

In our laboratory, we have recently derived and characterized a host of hESC lines from cloned human blastocysts or normal blastocysts [9-11]. In this report, we describe methods for the derivation of hESC lines, depending on the quality of blastocysts grown from cryopreserved $2 \mathrm{PN}$-stage embryos. These surplus embryos were originally donated for research purposes after prior approval from the institutional review board of the Seoul National University Hospital as well as written consent provided by donors who underwent in vitro fertilization (IVF).

\section{Quality of Blastocysts and hESC Derivation Methods}

In IVF programs, blastocyst quality is one of the most important factors in the determination of both implantation and pregnancy. There have been several reports that have compared the efficiency of implantation and pregnancy associated with highquality blastocysts possessing distinct and large ICMs versus poor-quality blastocysts [13-15]. Because hESCs can be derived from the ICM of the expanded blastocysts, not only blastocyst quality but also optimal ICM size and shape are crucial to this process. Therefore, we have used three different methods for the derivation of hESCs, depending on the morphology of the blastocysts and the appearance of their ICMs. We adopted the blastocyst grading system developed by the Cornell University (Ithaca, NY) program [16] and used it to rate the quality of our blastocysts before attempting hESC derivation (Fig. 1). In our $\mathrm{hESC}$ derivation process, good blastocysts, or those possessing large and distinct ICMs, (Fig. 1A), were subjected to the immunosurgical method. We used the partial-embryo culture method for expanded blastocysts with distinct but smaller ICMs (Fig. 1B). Finally, we opted for the use of the whole-embryo culture method in cases in which the blastocyst exhibited an indistinguishable trophectoderm and ICM (Fig. 1C).

\section{Immunosurgical Method}

In many laboratories, it has become common practice to isolate the ICM from expanded blastocysts via immunosurgery [12]. Our laboratory has also established hESC lines via a modified version of this technique in cases in which the blastocysts used possessed large and distinct ICMs [10].
Cryopreserved 2PN human embryos, which were provided by donations from the IVF clinic, were thawed and cultured for 5-7 days in G1.3, G2.3 media (Vitrolife, Gothenburg, Sweden, http:// www.vitrolife.com) until achieving expanded blastocyst stage. Each of these blastocysts was then washed twice (1 minute each) in medium I (knockout Dulbecco's modified Eagle's medium [DMEM] [Gibco-BRL/Invitrogen, Carlsbad, CA, http://www. invitrogen.com] supplemented with $10 \%$ fetal bovine serum [HyClone, Logan, UT, http://www.hyclone.com] or $20 \%$ knockout serum replacement [Gibco], 4 ng/ml human bFGF [Gibco], 2,000 $\mathrm{U} / \mathrm{ml}$ leukemia inhibitory factor [LIF] [Chemicon International, Temecula, CA, http://www.chemicon.com] [the LIF is added to the medium to establish the hESCs; after hESC derivation, the maintenance of the hESCs requires no further additions of LIF], $2 \mathrm{mM}$ L-glutamine, 1\% nonessential amino acid [NEAA] [Gibco], 0.1 mM $\beta$-mercaptoethanol [Sigma-Aldrich, St. Louis, http://www. sigmaaldrich.com], 50 units of penicillin, and $50 \mu \mathrm{g} / \mathrm{ml}$ of streptomycin [Gibco]). The blastocyst was then pipetted into a 50- $\mu$ l droplet of pronase (10 IU/ml) (Sigma-Aldrich) for 3-4 minutes to dissolve the zona pellucida (Fig. 2A), after which the enzyme-treated blastocyst was transferred to a 50- $\mu$ d droplet of medium I. A thin glass pipette was then used in the removal of the zona pellucida by lightly pipetting up and down, because the pronase treatment normally did not completely dissolve the zona pellucida. The blastocyst, after the complete removal of its zona pellucida, was washed approximately two to three times in a $50-\mu 1$ droplet of medium I. Anti-human whole-serum antibody (Sigma-Aldrich) and guinea

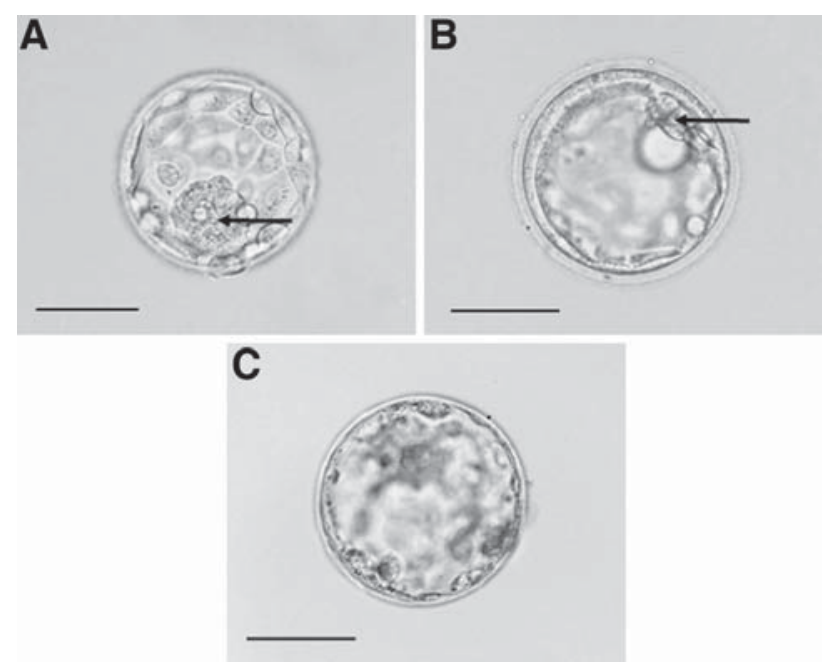

Figure 1.hESC derivation methods depending on blastocyst quality. hESC derivation techniques can be adopted according to the quality of the blastocyst. (A): Good blastocysts, which harbor large and distinct ICMs, were processed via the immunosurgical method. (B): Expanded blastocysts with small ICMs were processed via the partial-embryo culture method. (C): Blastocysts with poorly defined ICMs were processed via the whole-embryo culture method. The arrows indicate the ICM regions. Scale bar $=100 \mu \mathrm{m}$. Abbreviations: hESC, human embryonic stem cell; ICM, inner cell mass. 
pig complement (Sigma-Aldrich) were then added before the carrying out of the immunosurgery. The blastocyst was subjected to antibody complement treatment and washing on a tissue culture dish in 20- to 50- $\mu 1$ droplets using a Pasteur pipette. The pronasetreated blastocyst, after being rinsed in medium I, was transferred to a 20- $\mu$ d droplet of anti-human whole-serum antibody (undiluted solution) and incubated for 30 minutes at $37^{\circ} \mathrm{C}$ in an atmosphere containing $5 \% \mathrm{CO}_{2}$. The antibody-treated blastocyst was rinsed three additional times with a 50- $\mu$ l droplet of medium I to remove any residual antibody. The blastocyst was then transferred to a 20$\mu \mathrm{l}$ droplet of guinea pig complement, diluted with gelatin veronal buffer (1:4 dilution; Sigma-Aldrich), and incubated for 30 minutes at $37^{\circ} \mathrm{C}$ in an atmosphere containing $5 \% \mathrm{CO}_{2}$. The trophectoderm was lysed during this antibody complement reaction (Fig. 2B). The
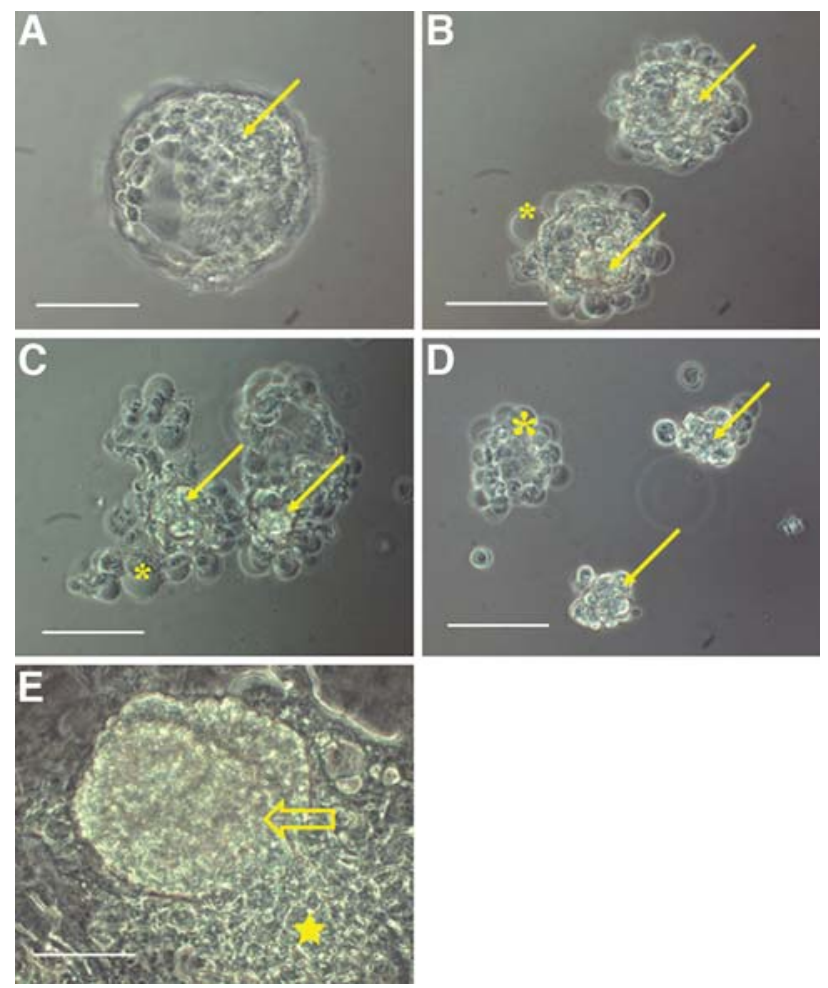

Figure 2. Immunosurgical method. (A): Good blastocysts, harboring large and distinct inner cell masses (ICMs), were treated with pronase to dissolve the zona pellucida. The blastocyst, now lacking its zona pellucida, was treated with anti-human whole antibody and guinea pig complement. (B): The trophectoderm was then lysed after the administration of antibody and complement treatment. (C): The lysed trophectoderm was gently separated from the ICM by repeated pipetting. (D): The ICM, completely separated from the trophectoderm, was then transferred to a layer of mitomycin C-treated STO feeder cells in $0.1 \%$ gelatin-coated tissue culture dishes. (E): After 7 days, the ICM, now surrounded by differentiated cells, had assumed a dome-like shape atop the feeder layer. The filled arrows indicate the ICM. The unfilled arrow indicates the ICM, having grown well after 7 days. The asterisks indicate the lysed trophectoderm. The star indicates the cells differentiated from the ICM. (B-D): Two different blastocysts with ICM and lysed trophectoderm. Scale bar $=100 \mu \mathrm{m}$. blastocyst, with its lysed trophectoderm, was then transferred to a 50- $\mu 1$ droplet of medium I and rinsed three times to remove any residual complement. A Pasteur pipette was then carefully produced, such that its diameter was slightly larger than the observed ICM. This pipette was then used to repeatedly pipette the blastocyst with lysed trophectoderm to separate the trophectoderm from the ICM (Fig. 2C). The isolated ICM (Fig. 2D) was then cultured on a layer of mitomycin $\mathrm{C}$-treated STO feeder cells (American Type Culture Collection, Manassas, VA, http://www.atcc.org) in a $0.1 \%$ gelatin (Sigma-Aldrich)-coated tissue culture dish with medium I. Two days later, the attachment of the ICM to the feeder layer was verified and one half of the medium was replaced with fresh medium I. Beginning on the third day, the quality of the ICM growth was monitored continuously under a microscope and the medium was replaced daily. A well-progressed ICM had attached to the STO feeder layer and had expanded to a diameter of approximately $150 \mu \mathrm{m}$ in 7 days. The ICM had formed a domelike structure atop the STO feeder layer (Fig. 2E). We used a finely drawn-out Pasteur pipette to cut out the 1-week-old ICM dome. The entire ICM dome structure was then transferred to a fresh STO feeder layer and incubated with medium II (DMEM/F12 [Gibco] media supplemented with $20 \%$ knockout serum replacement, 4 $\mathrm{ng} / \mathrm{ml}$ human basic fibroblast growth factor [bFGF], 2,000 U/ml LIF, $1 \%$ NEAA, $0.1 \% \beta$-mercaptoethanol, 50 units of penicillin, and $50 \mu \mathrm{g} / \mathrm{ml}$ streptomycin). The ICM, after being transferred to
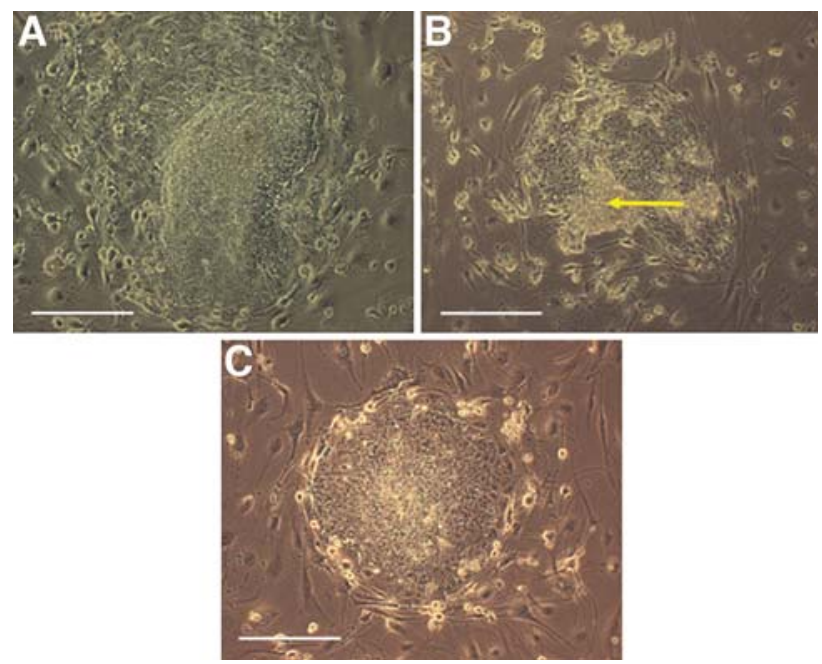

Figure 3. Various colony morphologies developed from first passaged inner cell mass. (A): Compact cells surrounded by differentiated cells. Only the compact cells were selected and were then continuously passaged. Colony morphology from the 4-day culture after the first passage. (B): The attached cells exhibited human embryonic stem cell (hESC)-like morphology in a colony. The unattached cells (arrow) were then removed when the medium was exchanged, and the attached cells were split and passaged. Colony morphology from the culture 2 days after the first passage. (C): A colony exhibiting the characteristic hESC colony-like morphology. Colony morphology from the culture 2 days after the first passage. Scale bar $=200 \mu \mathrm{m}$. 
a new dish, tended to manifest with diverse colony morphologies (Fig. 3). The observed cell morphologies in these cases often were not reminiscent of those seen in genuine hESC colonies. Many differentiated cells were observed to have formed around the compact cells (Fig. 3A). When the compact cells were isolated and passaged, we noted the formation of characteristic hESC-like colonies in subsequent passages. As is shown in Figures $3 \mathrm{~B}$ and 3C, the hESC-like colonies also could be observed with a high ratio of nucleus to cytoplasm. Among the observed colonies, the portions that evidenced hESC-like morphology (Fig. 3B) or hESC-like colonies (Fig. 3C) were divided into small clumps and then passaged to acquire hESC colonies [17].

\section{Partial-Embryo Culture Method}

The partial-embryo culture method was used in cases in which an expanded blastocyst developed but exhibited a smaller ICM than was seen in the good blastocyst cases (Fig. 1B). These blastocysts were determined to harbor a higher risk of losing their ICMs when processed via immunosurgery.

Therefore, we devised the partial-embryo culture method (Fig. 4) via modification of the whole-embryo culture method (see below for whole-embryo culture), seeking to improve the weak points of this technique. The partial-embryo culture method, unlike the whole-embryo culture method, alleviates the issue of
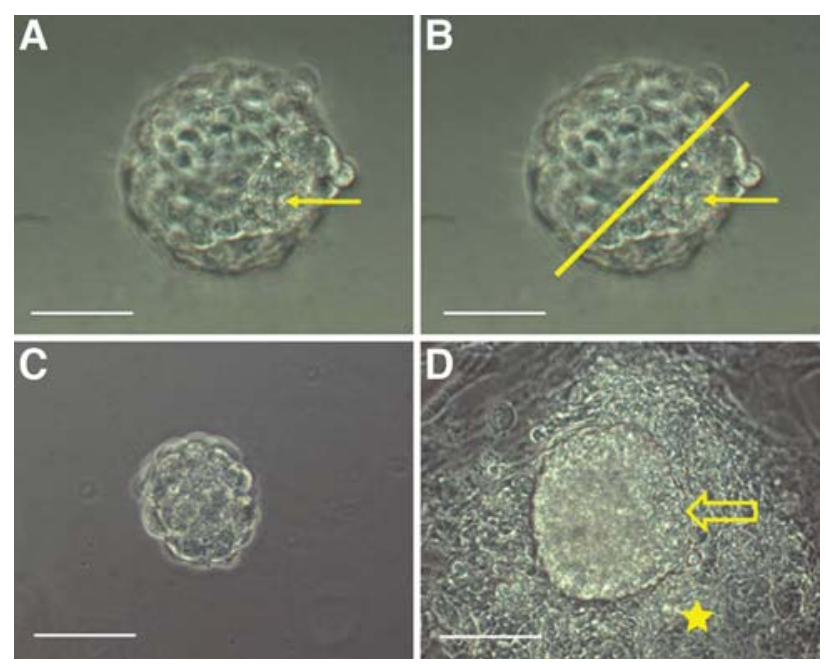

Figure 4. Partial-embryo culture method. (A): The expanded blastocyst was treated with pronase to dissolve the zona pellucida. (B): The small region in which the inner cell mass (ICM) was located was surgically isolated with a finely drawn glass pipette along the indicated line. The portion of the embryo in which the ICM, covered by the remaining trophectoderm, was located $(\mathbf{C})$ was transferred to a layer of mitomycin C-treated STO feeder cells in $0.1 \%$ gelatin-coated tissue culture dishes. (D): After 7 days, the ICM grew to assume a large dome-like shape, and the residual trophectoderm and differentiated cells surrounded the dome-like ICM region. The filled arrows indicate the ICM. The unfilled arrow indicates the well-grown dome-like ICM. The star indicates the trophectoderm and differentiated cells. Scale bar $=100 \mu \mathrm{m}$. trophectoderm overgrowth by virtue of the surgical isolation of the region in which the ICM is located. This surgical procedure was determined to significantly reduce the risk of trophectoderm overgrowth, which tends to inhibit the growth of the ICM.

Cryopreserved 2PN embryos were cultured in G1.3, G2.3 media until expanded blastocyst stage was achieved, and some of these blastocysts exhibited normal morphologies but smaller ICMs (Fig. 1B). These expanded blastocysts were treated with pronase to dissolve their zona pellucida (Fig. 4A), and the region in which the ICM was located was surgically isolated, with an eye toward excluding as much trophectoderm as possible, using a finely drawn glass pipette (Fig. 4B). After this mechanical isolation, the ICM remained enveloped in only a small layer of trophectoderm (Fig. 4C). This partial embryo, comprised of ICM and trophectoderm, was then transferred to a mitomycin $\mathrm{C}$-treated STO feeder layer and was cultured in medium I. Two days after the transfer, we noted the status of the partial embryo under a microscope to monitor cell attachment. Half of the old medium was carefully replaced with fresh medium. On the third day of the culture, the trophectoderm began to spread on the culture dish. The status of the cells was checked on a daily basis, and the medium was also replaced on a daily basis. After 7 days, the ICM assumed the characteristic dome-like shape and the trophectoderm was observed to surround the ICM (Fig. 4D). The ICM dome structure was then isolated, again using a finely drawn glass Pasteur pipette. The selected ICM region was then transferred to a freshly prepared STO feeder layer, and medium II was used to begin culturing these cells. Two days after the transfer, the cells were again observed under a microscope and the medium was exchanged. Cell growth was subsequently monitored on a daily basis. Diverse colony morphologies were observed in a manner similar to that seen in the previously described derivation method (Fig. 3). Only the colonies that exhibited distinct hESC-like morphology (Fig. 3C) or compact cell morphology (Fig. 3A) and portions of colonies exhibiting hESC-like morphology (Fig. 3B) were selected, mechanically divided into small clumps, and transferred to new dishes. After several passages, remnants of the trophectoderm were no longer observed.

\section{Whole-Embryo Culture Method}

We used this technique to isolate the ICM and to establish hESC lines in cases in which the blastocyst lacked a distinct ICM (Fig. 1C). Because we were unable to visualize the ICM in these blastocysts, neither the immunosurgical method nor the partial-embryo culture method was a suitable method for the isolation of the ICM. The cryopreserved 2PN embryos were cultured in G1.3, G2.3 media until expanded blastocyst stage was achieved. Among these blastocysts, those with indistinct or invisible ICMs were treated with pronase to dissolve the zona pellucida (Fig. 5A). The whole embryo, with its zona pellucida having been completely dissolved, was then plated on a mitomycin C-treated STO feeder 
layer. Two days after this plating, the attachment of the whole embryo to the feeder layer was confirmed and half of the volume of the old medium was replaced with fresh medium. Thereafter, the status of the whole embryo attached to the feeder layer was observed on a daily basis, and the medium was also replaced with fresh medium on a daily basis. The trophectoderm layer began to expand on the third day of culturing. On day 7, the ICM-like cells formed a dome structure, surrounded by trophectoderm (Fig. 5B). At this point, we used a finely drawn Pasteur pipette to push the trophectoderm away from the ICM dome. The thin layer of cells atop the ICM dome was removed, after which the ICM was transferred to a freshly prepared STO feeder layer. Medium II was then used for the passaging of these cells. Two days after the transfer, the cells were observed under a microscope and the medium was replenished, as previously described. The cells were monitored and cell growth was verified on a daily basis. In cases in which the ICM grew successfully, the trophectoderm disappeared after repeated passages and we noted the formation of colonies with characteristic hESC morphologies. The whole-embryo culture tends to manifest an abundance of both trophectoderm and differentiated cells, and the isolation of hESC-like colonies from differentiated cells requires a great deal of care and caution. However, the poor quality of the blastocysts often results in failures in the attachment of the ICM to the dish.
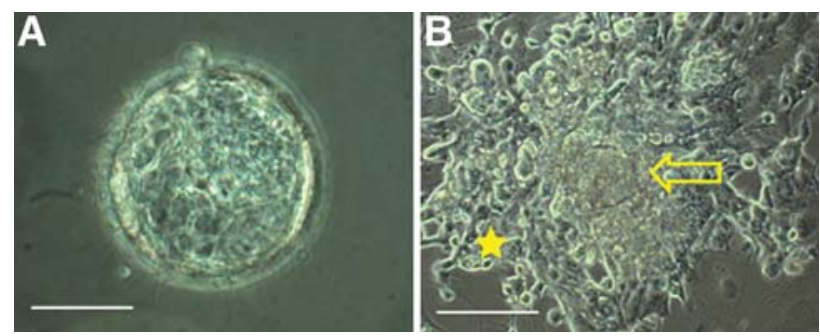

Figure 5. Whole-embryo culture method. The blastocyst in which no distinct inner cell mass (ICM) could be discerned was treated with pronase to dissolve the zona pellucida (A) and then directly transferred to a layer of mitomycin C-treated STO feeder cells. (B): After 7 days, the ICM, covered with the trophectoderm, grew to assume a large dome-like shape, and the trophectoderm and differentiated cells were observed to surround the dome-like ICM region. The unfilled arrow indicates the dome-shaped ICM. The star indicates the trophectoderm and the differentiated cells. Scale bar $=(\mathbf{A}) 100$ $\mu \mathrm{m}$ and (B) $200 \mu \mathrm{m}$.
The whole-embryo culture method seems to be quite advantageous with regard to the establishment of animal pathogen-free hESCs because of the absence of animal antibodies and complements in its ICM isolation step. This procedure, however, runs a much greater risk of trophectoderm overgrowth than the other two methods because the entire trophectoderm is cultured along with the ICM. These trophectodermal cells often hinder the growth of the ICM during the culture period and render the establishment of hESC lines rather problematic [18, 19]. It was recently reported that several ESC lines were derived from cloned human embryos using whole-embryo culture method [11]. This method may be beneficial in the case of derivation of hESCs from cloned embryos produced by a process of nuclear transfer.

\section{Conclusion}

In this study, we have reported and described three distinct methods for the derivation of hESCs based on the quality of the expanded blastocysts. These methods are referred to as the immunosurgical, partial-embryo culture, and whole-embryo culture methods.

We established 13 hESC lines from 47 blastocysts using each of the three aforementioned derivation methods (Table 1). The establishment of hESC lines from high-quality blastocysts possessing large and distinguishable ICMs was determined to be slightly more successful when the immunosurgical method was used (four cell lines established from seven blastocysts) than when the partial-embryo culture method was used (two cell lines established from five blastocysts). However, in cases in which the expanded blastocyst exhibited normal morphology but a somewhat smaller ICM, the partial-embryo culture method (four cell lines from eight blastocysts) proved rather more effective than the immunosurgical method (two cell lines from 12 blastocysts) with regard to the establishment of hESC lines. However, neither the immunosurgical nor the partial-embryo culture method was particularly successful in the establishment of hESC lines from blastocysts in which the ICM and trophectoderm were poorly defined. Using the whole-embryo culture method, we were able to establish only one line from nine such blastocysts.

The first method, immunosurgery, is commonly practiced in many laboratories. This method is quite efficient in the establishment of a cell line from a good-looking expanded blastocyst, which harbors a large and distinct ICM (Figs. 1A, 2). However,

Table 1. Derivation of human embryonic stem cells, depending on ICM size and morphology

\begin{tabular}{lllll}
\hline & \multicolumn{3}{c}{ Size and morphology of ICM } & Total \\
\cline { 2 - 5 } Derivation method & Large and distinct & Small and distinct & Not visible & $6 / 22(27.3 \%)^{\mathrm{a}}$ \\
\hline Immunosurgical method & $4 / 7(57.1 \%)^{\mathrm{a}}$ & $2 / 12(16.7 \%)^{\mathrm{a}}$ & $0 / 3(0 \%)^{\mathrm{a}}$ & $6 / 16(37.5 \%)^{\mathrm{a}}$ \\
Partial-embryo culture method & $2 / 5(40 \%)^{\mathrm{a}}$ & $4 / 8(50 \%)^{\mathrm{a}}$ & $0 / 3(0 \%)^{\mathrm{a}}$ & $1 / 9(11.1 \%)^{\mathrm{a}}$ \\
Whole-embryo culture method & Not Done & Not Done & $1 / 9(11.1 \%)^{\mathrm{a}}$ & $13 / 47(27.7 \%)^{\mathrm{a}}$ \\
Total & $6 / 12(50 \%)^{\mathrm{a}}$ & $6 / 20(30 \%)^{\mathrm{a}}$ & $1 / 15(6.7 \%)^{\mathrm{a}}$ & \\
\hline
\end{tabular}

aPercentage does not indicate statistical meaningfulness due to a limitation on the number of the human embryos used in this study. Abbreviation: ICM, inner cell mass. 
among blastocysts, there are also ones with smaller or indistinct ICMs. In these cases, the immunosurgical method tends to result in the loss of the ICM when the lysed trophectodermal layer is removed during the ICM isolation step. The possibility that the hESC will become contaminated with animal pathogens during the antibody and complement treatment is also an issue that concerns many researchers. To address and overcome these limitations, whole- or partial-embryo culture methods can be used as alternatives to immunosurgery. When dealing with blastocysts harboring indistinct ICMs, the whole-embryo culture method is most frequently used. This technique establishes an animal pathogen-free hESC line via the seeding of the blastocyst only after pronase treatment has been administered. However, because the blastocyst is treated only with pronase, the trophectoderm still remains in this case. The trophectoderm can then impede the growth of the ICM and generally complicate hESC cultivation. The partial-embryo culture method addresses this inherent shortcoming of the whole-embryo culture method, while retaining the decided advantage of being free of possible contamination with animal pathogens. The partial-embryo culture method partially removes the trophecto-

\section{REFERENCES}

1 Evans MJ, Kaufman MH. Establishment in culture of pluripotential cells from mouse embryos. Nature 1981;292:154-156.

2 Martin GR. Isolation of a pluripotent cell line from early mouse embryos cultured in medium conditioned by teratocarcinoma stem cells. Proc Natl Acad Sci US A 1981;78:7634-7638.

3 Thomson JA, Kalishman J, Golos TG et al. Isolation of a primate embryonic stem cell line. Proc Natl Acad Sci US A 1995;92:7844-7848.

4 Thomson JA, Itskovitz-Eldor J, Shapiro SS et al. Embryonic stem cell lines derived from human blastocysts. Science 1998;282:1145-1147.

5 Reubinoff BE, Pera MF, Fong CY et al. Embryonic stem cell lines from human blastocysts: somatic differentiation in vitro. Nat Biotechnol 2000;18:399-404.

6 Park JH, Kim SJ, Oh EJ et al. Establishment and maintenance of human embryonic stem cells on STO, a permanently growing cell line. Biol Reprod 2003;69:2007-2014.

7 Stojkovic M, Lako M, Stojkovic P et al. Derivation of human embryonic stem cells from day-8 blastocysts recovered after three-step in vitro culture. STEM CELLS 2004;22:790-797.

8 Cowan CA, Klimanskaya I, McMahon J et al. Derivation of embryonic stem-cell lines from human blastocysts. N Engl J Med 2004;350:13531356.

9 Hwang WS, Ryu YJ, Park JH et al. Evidence of a pluripotent human embryonic stem cell line derived from a cloned blastocyst. Science 2004;303:1669-1674. dermal layer by virtue of the mechanical separation of the ICM region from as much of the trophectoderm as possible. This method allows for the efficient derivation of hESC lines from blastocysts with small ICMs. In our experience, this method is also applicable to blastocysts in which the ICM is large and distinct, and the efficiency of this method is comparable to that of the immunosurgical method (Table 1). To obtain animal pathogen-free hESC lines, the partial-embryo culture method may be more appropriate, especially for clinical use in humans. The derivation techniques presented herein should provide useful tools toward the establishment of hESC lines, depending on the quality of the blastocyst.

\section{ACKNOWLEDGMENTS}

This research was supported by grants (SC1020, SC2140, and SC2040) from the Stem Cell Research Center of the 21st Century Frontier Research Program, which is funded by the Ministry of Science and Technology, Republic of Korea.

\section{Disclosures}

The authors indicate no potential conflicts of interest.
10 Oh SK, Kim HS, Ahn HJ et al. Derivation and characterization of new human embryonic stem cell lines, SNUhES1, SNUhES2, and SNUhES3. STEM CELLS 2005;23:211-219.

11 Hwang WS, Roh SI, Lee BC et al. Patient-specific embryonic stem cells derived from human SCNT blastocysts. Science 2005;308:1777-1783.

12 Solter D, Knowles BB. Immunosurgery of mouse blastocyst. Proc Natl Acad Sci U S A 1975;72:5099-5102.

13 Dokras A, Sargent IL, Barlow DH. Human blastocyst grading: an indicator of developmental potential? Hum Reprod 1993;8:2119-2127.

14 Richter KS, Harris DC, Daneshmand ST et al. Quantitative grading of a human blastocyst: optimal inner cell mass size and shape. Fertil Steril 2001;76:1157-1167.

15 Kovacic B, Vlaisavljevic V, Reljic M et al. Developmental capacity of different morphological types of day 5 human morulae and blastocysts. Reprod Biomed Online 2004;8:687-694.

16 Veeck LL, Zaninovic N. Human blastocysts in vitro. In: Veeck LL, Zaninovic N, eds. An Atlas of Human Blastocysts. New York: The Parthenon Publishing Group, 2003:99-137.

17 Oh SK, Kim HS, Park YB et al. Methods for expansion of human embryonic stem cells. STEM CELLS 2005;23:605-609.

18 Pickering SJ, Braude PR, Patel M et al. Preimplantation genetic diagnosis as a novel source of embryos for stem cell research. Reprod Biomed Online 2003;7:353-364.

19 Bongso A, Fong CY, Ng SC et al. Isolation and culture of inner cell mass cells from human blastocysts. Hum Reprod 1994;9:2110-2117. 